\title{
Diagnostic Value of Serum IncRNA HOTAIR Combined with Galectin-3 in Benign and Papillary Thyroid Carcinoma
}

\author{
$\mathrm{Li} \mathrm{Li}^{1, *}$ \\ Jianjun Wang ${ }^{1, *}$ \\ Zhongquan $\mathrm{Li}^{2}$ \\ Shuang Qiu' \\ Junyu Cao' \\ Yuan Zhao' \\ Zhenfan Huang' \\ Jie $\mathrm{He}^{\text {l }}$ \\ Feipeng Luo ${ }^{3}$ \\ Kunxian Yang \\ 'Department of Breast and Thyroid \\ Surgery, The First People's Hospital of \\ Yunnan Province (Kunhua Hospital \\ Affiliated to Kunming University of \\ Science and Technology), Kunming, \\ Yunnan, 650032, People's Republic of \\ China; ${ }^{2}$ Department of Anesthesiology, \\ Yunnan General Hospital of Armed \\ Police, Kunming, Yunnan, 650032, \\ People's Republic of China; ${ }^{3}$ Department \\ of Cardiothoracic Surgery, The Second \\ People's Hospital of Baoshan City, \\ Kunming, Yunnan, 650032, People's \\ Republic of China
}

*These authors contributed equally to this work
Purpose: Papillary thyroid carcinoma (PTC) is the most common type of thyroid cancer. LncRNA HOTAIR (HOx Transcript AntIsense RNA) and Galectin-3 are involved in PTC. This study explored the clinical effect of lncRNA HOTAIR/Galectin-3 on PTC patients.

Methods: Subjects were assigned into PTC (160 cases) and benign thyroid tumor groups (150 cases). Fasting peripheral venous blood was collected. LncRNA HOTAIR/Galectin-3 expressions in serum were detected. Subjects were assigned into HOTAIR/Glactin-3 high/ low expression groups and their correlation with age, gender, BMI, tumor size, pathological stage, TSH, TPO-Ab, and TG-Ab in PTC was analyzed. Receiver operating characteristic (ROC) curve was conducted on diagnostic efficacy of HOTAIR or/and Galectin-3. The difference of area under the curve (AUC) was compared and analyzed.

Results: HOTAIR and Glactin-3 were higher in PTC group and correlated with tumor pathological stage. Higher HOTAIR/Glactin-3 expression indicated a more advanced TNM stage. LncRNA HOTAIR was positively correlated with TPO-Ab and TG-Ab. AUC of HOTAIR for PTC diagnosis was 0.895 , with $96.00 \%$ specificity and $80.63 \%$ sensitivity. AUC of Glactin-3 for PTC diagnosis was 0.817 , with $66.67 \%$ specificity and $78.75 \%$ sensitivity. AUC of HOTAIR combining with Glactin-3 for PTC diagnosis was 0.969 with $96.00 \%$ specificity and $87.50 \%$ sensitivity. AUC of lncRNA HOTAIR was higher than that of Glactin-3, while AUC of the combination was higher than that of lncRNA HOTAIR or Glactin-3.

Conclusion: LncRNA HOTAIR and Glactin-3 were highly expressed in PTC. The combination detection of IncRNA HOTAIR/Glactin-3 had higher diagnostic efficiency on the differential diagnosis of benign thyroid tumor and PTC.

Keywords: papillary thyroid carcinoma, LncRNA HOTAIR, Glactin-3, combination detection, benign thyroid tumor, ROC curve analysis, diagnostic efficiency, TPO-Ab, PTC

\section{Introduction}

Thyroid cancer is a common malignancy in the endocrine system, which has a sharply and continuously increasing incidence worldwide in the last decades. ${ }^{1,2}$ Histologically, thyroid cancer is mainly divided into three types, papillary thyroid carcinoma (PTC), anaplastic thyroid cancer, and follicular thyroid cancer. ${ }^{3-6}$ PTC is the most prevalent histological form of the differentiated malignant tumor of the thyroid, which is identified as the least aggressive form of thyroid tumor. ${ }^{7}$ Generally, most patients show a favorable postoperative prognosis as a welldifferentiated thyroid neoplasm and the PTC mortality rate is about $11-17 \%{ }^{8}$
Correspondence: Kunxian Yang Department of Breast and Thyroid Surgery, The First People's Hospital of Yunnan Province (Kunhua Hospital Affiliated to Kunming University of Science and Technology), No. 157, Jinbi Road, Xishan District, Kunming, Yunnan, 650032, People's Republic of China Tel +86-087l-63627I84

Email kunxy0125@I63.com 
However, the clinical course of PTC is not obvious. Typical PTC is characterized by papillary structure and unique nuclear morphology, such as vitreous nucleus, nuclear groove, and intranuclear inclusion body; however, it is difficult to distinguish PTC from benign thyroid lesions such as nodular goiter, Hashimoto's thyroiditis and papillary adenoma with papillary growth; at present, the differential diagnosis markers of PTC and benign thyroid tumor with papillary hyperplasia are CK19/ Galectin-3/HBME1, but their sensitivity and specificity are relatively low, so that their clinical application is limited. Therefore, the differential diagnosis of PTC is still difficult. ${ }^{9}$ With the development of molecular biology and the emergence of various biomarkers, many researchers try to find new molecular biomarkers for the early diagnosis and prognosis evaluation of PTC. Therefore, it is of vital importance to identify new diagnostic and prognostic biomarkers for PTC patients.

Long noncoding RNAs (lncRNAs) are a kind of nontranslating RNAs with a length of more than 200 nucleotides, which have stability in serum and other biological fluids and play a role in various diseases and tissue specificity ${ }^{10}$ LncRNAs regulate the expression of genes and are involved in a variety of biological processes, including cell migration, invasion, proliferation, apoptosis, and cell cycle, and are aberrantly expressed in the process of tumorigenesis, especially in PTC. ${ }^{11}$ HOx Transcript AntIsense RNA (HOTAIR) has been reported to be overexpressed in serum of PTC patients with lymph node metastasis. ${ }^{12}$ Notably, lncRNA HOTAIR plays a role in the malignant biological behaviors of PTC through regulating the miR-488-5p/NUP205 axis. ${ }^{13}$ LncRNA-HOTAIR in tissue samples of PTC patients has been recommended for the prognosis of PTC. ${ }^{14}$ However, the clinical diagnostic value of serum lncRNA-HOTAIR for PTC has not been reported.

Galectin-3 is a carbohydrate-binding protein, which plays a role in the processes related to the progression and metastasis of tumors including malignant cell behaviors, dynamic cellular transformation, and heterotypic/ homotypic aggregation. ${ }^{15}$ Galectin-3 has been identified to be a recognized tissue marker of PTC and has good specificity in differentiating thyroid cancer, so it can be used as a histological marker of thyroid cancer, which is not expressed by normal thyroid cells and it has good specificity in the differential diagnosis of thyroid cancer. ${ }^{16}$ However, there are few studies on the value of Galectin-3 in the diagnosis and prognosis of PTC. ${ }^{17,18}$
Whether serum Galectin-3 can be used as a biomarker for the diagnosis of PTC remains to be studied.

Studies have reported that most lncRNAs can act as microRNA (miRNA) sponges through the competitive endogenous RNA (ceRNA) network, thus upregulating the expression of downstream mRNA; without exception, IncRNA HOTAIR has also been reported to play a role as miR-206 ceRNA in neuroblastoma, ovarian cancer and other diseases. ${ }^{19-21}$ miR-206 has been documented to be significantly downregulated in PTC. ${ }^{22}$ Moreover, the RNAInter database showed that miR-206 had a targeted binding relationship with Galectin-3 (http://www.rnasociety.org/rnainter/php mysql/more.php? raid= RR03364696). Based on the above analysis, we speculated that IncRNA HOTAIR might play a role in PTC by regulating Galectin-3 expression through the ceRNA mechanism. However, there is no domestic and foreign report at present on the diagnostic value of serum lncRNA HOTAIR and its combination with Galectin-3 in differentiating benign thyroid tumors from PTC. This study set out to investigate the diagnostic efficacy of lncRNA HOTAIR combined with Glactin-3 in PTC, to provide some reference for the diagnostic value of differentiating benign thyroid tumors from PTC.

\section{Materials and Methods Ethics Statement}

The clinical study protocol was designed and implemented following the relevant provisions of the Helsinki Declaration on the protection of the rights and interests of subjects and the experiments were authorized by the academic ethics committee of The First People's Hospital of Yunnan Province (Kunhua Hospital Affiliated to Kunming University of Science and Technology). All procedures were strictly implemented according to the code of ethics. All subjects involved were fully informed of the objective of the study and signed the informed consent before sampling.

\section{Study Subjects and Sample Collection}

The clinical data of 310 patients with thyroid tumor who accepted surgical resection in The First People's Hospital of Yunnan Province (Kunhua Hospital Affiliated to Kunming University of Science and Technology) from December 2015 to December 2020 were collected. According to the pathology after the operation, the subjects were assigned to the PTC group (160 cases) and the benign thyroid tumor group (control group, 150 cases, including 47 
cases of hyperplastic nodules, 37 cases of benign papillary hyperplasia, and 66 cases of thyroid adenoma). Inclusion criteria were as follows: 1) The clinical data were complete; 2) No special treatment (such as radiotherapy, chemotherapy, hormone replacement therapy, etc.) was given before operation; 3) The patients were confirmed to be PTC or benign thyroid tumor according to postoperative pathology; and 4) There was a clear pathological stage. Exclusion criteria were as follows: 1) Patients were complicated with other cancers; and 2) Patients were complicated with adrenal diseases. Tumor-node-metastasis (TNM) staging standard followed the 8th edition of the American Joint Committee on Cancer classification system. ${ }^{23}$ After admission, $2 \mathrm{~mL}$ fasting peripheral venous blood was collected immediately and centrifuged at $2000 \mathrm{~g}$ at $4^{\circ} \mathrm{C}$ for $10 \mathrm{~min}$. The supernatant was transferred to the Eppendorf (EP) tube and stored at $-80^{\circ} \mathrm{C}$. The samples were not in any freeze-thaw cycles before this study.

\section{Clinicopathological Parameters Detection}

The levels of serum high-sensitive thyroid-stimulating hormone (TSH), thyroid peroxidase antibody (TPO-Ab), and thyroglobulin antibody (TG-Ab) were detected using the Beckman DXI800 chemiluminescence method.

\section{Total RNA Extraction and Reverse Transcription Quantitative Polymerase Chain Reaction (RT-qPCR)}

The serum total RNA was extracted using TRIzol reagent (Invitrogen, Carlsbad, CA, USA) And reverse transcribed into cDNA using PrimeScript RT reagent kit (Takara Biotechnology Inc., Dalian, China). The qPCR was performed using SYBR $^{\circledR}$ Premix Ex TaqTM II (Takara Biotechnology Inc.) on the ABI7900HT fast PCR real-time system (Applied Biosystems, Foster city, CA, USA). The reaction conditions consisted of pre-denaturation at $95^{\circ} \mathrm{C}$ for 10 min and 40 cycles of denaturation at $95^{\circ} \mathrm{C}$ for $10 \mathrm{~s}$, annealing at $60^{\circ} \mathrm{C}$ for $20 \mathrm{~s}$ and extending at $72^{\circ} \mathrm{C}$ for 34 s. With U6 as an internal parameter, the relative expression of the gene was calculated based on the
$2^{-\Delta \Delta \mathrm{Ct}}$ method. ${ }^{24}$ The primer sequences synthesized by Sangon Biotech Co., Ltd., (Shanghai, China) are shown in Table 1.

\section{Enzyme-Linked Immunosorbent Assay (ELISA)}

The Galectin-3 content in serum was detected using an ELISA kit (ZN2192, Biolab, Beijing, China). Each well of the enzyme-coated plate was added with $10 \mu \mathrm{L}$ serum samples and $40 \mu \mathrm{L}$ sample diluents, and then the plate was sealed and bathed at $37^{\circ} \mathrm{C}$ for $30 \mathrm{~min}$. After 5 times of washing, the reaction plate was added with $50 \mu \mathrm{L}$ enzyme labeling reagent, and then sealed and bathed at $37^{\circ} \mathrm{C}$ for $30 \mathrm{~min}$. After washing, the reaction plate was added with $50 \mu \mathrm{L}$ developer $\mathrm{A}$ and developer $\mathrm{B}$. The samples were mixed well and placed at $37^{\circ} \mathrm{C}$ in the dark for $15 \mathrm{~min}$. Each well was added with $50 \mu \mathrm{L}$ termination solutions to terminate the reaction. The color was altered from blue to yellow at this time. Finally, the absorbance at a wavelength of $450 \mathrm{~nm}$ was measured using a Multiskan ${ }^{\mathrm{TM}}$ FC microplate (Thermo Fisher Scientific, Inc., Waltham, MA, USA). Serum Galectin3 level was calculated using the standard curve.

\section{Statistical Analysis}

SPSS 21.0 (IBM Corp. Armonk, NY, USA), GraphPad Prism 8 (GraphPad Software), and MedCalc ${ }^{\circledR}$ version 15.0 (MedCalc Software Ltd, Ostend, Belgium) statistical software were used for data analysis and mapping. The variable data were expressed as mean \pm standard deviation (continuous variable), or counting and percentage (categorical variable). The $T$ test or $\chi^{2}$ test was applied for comparison among groups. Receiver operating characteristic (ROC) curve was used to analyze the diagnostic value of IncRNA HOTAIR, Glactin-3, and their combination in PTC. Binary logistic regression analysis was used to calculate the joint predictor and the area under the ROC curve (AUC) was analyzed using MedCalc-Comparison of ROC curves. A value of $P<0.05$ indicated statistical significance.

Table I Primer Sequence

\begin{tabular}{|l|l|l|}
\hline Gene & Forward 5'-3' & Reverse 5'-3' \\
\hline LnCRNA HOTAIR & GGTCTTGCCTCCTCTCTGTG & CTCTGGCCAGGAAAAGAGTG \\
GAPDH & CTCAGACACCATGGGGAAGGTGA & ATGATCTTGAGGCTGTTGTCATA \\
\hline
\end{tabular}




\section{Results}

\section{Comparison of Baseline Data Between} PTC Patients and Benign Thyroid Tumor Patients

A total of 310 subjects were included in this study, including 150 cases in the benign thyroid tumor group and 160 cases in the PTC group. Among 160 cases of PTC patients, 85 patients had a tumor diameter of $2 \mathrm{~cm}$ or more (53.13\%), 88 patients had a single lesion $(55.00 \%), 72$ patients had multiple lesions (45.00\%); 117 cases were in the TNM stage I-II (73.13\%) and 43 cases were in stage III-IV (26.87\%); 94 patients had lymph node metastasis (58.75\%), and 11 patients had distant metastasis (6.88\%). The clinical features of the PTC group and benign thyroid tumor group were compared and analyzed. There was no significant difference in age, gender, body mass index (BMI), and TSH level between the two groups. The levels of TPO-Ab and TG-Ab in the PTC group were significantly higher than those in the benign thyroid tumor group (Table 2).

\section{LncRNA HOTAIR and Glactin-3 Were Highly Expressed in PTC}

The serum expression of IncRNA HOTAIR in the PTC group and benign thyroid tumor group was detected using RT-qPCR. The serum expression of lncRNA HOTAIR in PTC patients was significantly higher than that in patients with benign thyroid tumor $(\mathrm{t}=15.47$, $P<0.01$ ) (Figure 1A). The level of Galectin-3 in serum was detected using ELISA. The serum Galectin-3 level in PTC patients was also higher than that in patients with benign thyroid tumor $(\mathrm{t}=11.71, P<0.01)$ (Figure 1B).

\section{Relationship Between IncRNA HOTAIR/ Glactin-3 and Clinical Baseline Characteristics}

To further study the correlation between IncRNA HOTAIR/Glactin-3 and PTC, PTC patients were assigned into a high expression group and a low expression group according to the median expression of IncRNA HOTAIR or Glactin-3, and the correlation analysis was performed with the clinical indexes of PTC patients. The results showed that patients in the lncRNA HOTAIR high expression group had larger tumor diameter, more advanced TNM stage and higher $\mathrm{T}$ stage, more lymph
Table 2 Comparison of Baseline Data Between PTC Patients and Benign Thyroid Tumor Patients

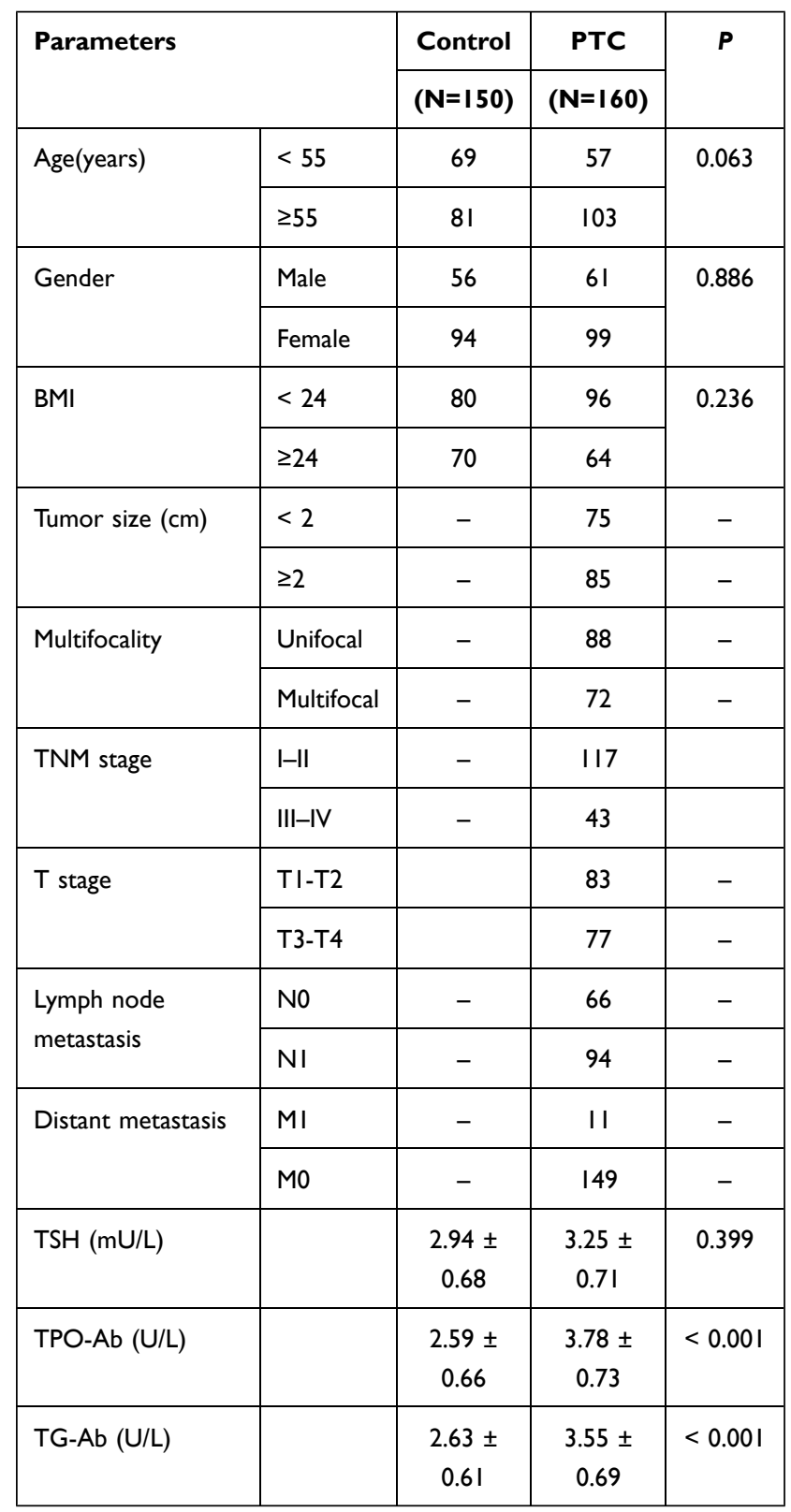

node metastasis, and higher TPO-Ab and TG-Ab expressions (all $P<0.05$ ), but with no correlation with age, gender, BMI, multifocality, distant metastasis and TSH expression (all $P>0.05$ ) (Table 3). Patients in the Glactin-3 high expression group were associated with larger tumor diameter, more advanced TNM stage, and higher $\mathrm{T}$ stage, more lymph node metastasis (all $P<$ 0.05 ), but was not related to age, gender, BMI, multifocality, distant metastasis, TSH, TPO-Ab and TG-Ab expressions (all $P>0.05$ ) (Table 4). 
A

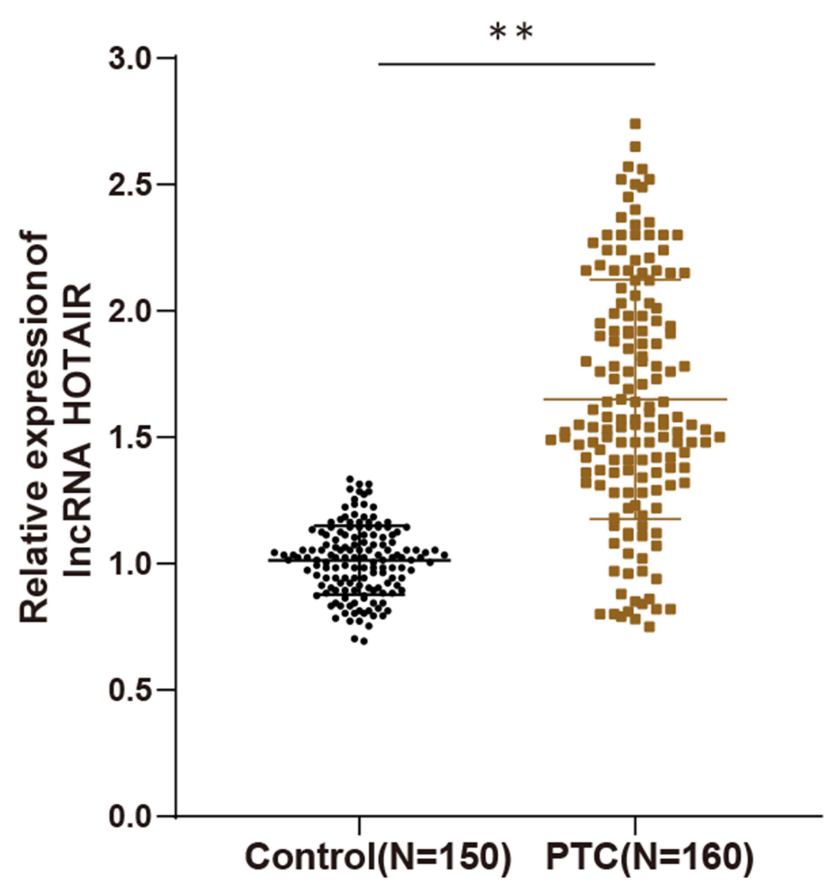

B

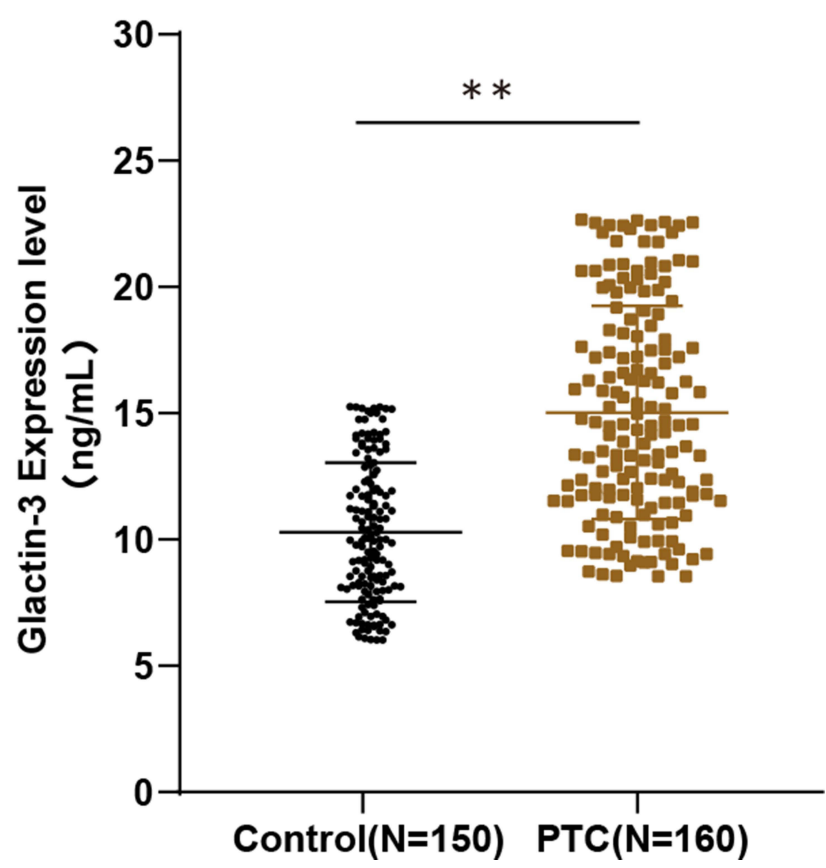

Figure I LncRNA HOTAIR and Glactin-3 were highly expressed in PTC. Fasting peripheral venous blood was collected immediately after patient admission. (A) LncRNA HOTAIR expression in serum of PTC group and benign thyroid tumor group was detected by RT-qPCR; (B) Galectin-3 expression in serum was detected using ELISA. The data were expressed as mean \pm standard deviation. $T$ test was used for comparison among groups. $* * P<0.0$ I.

\section{LncRNA HOTAIR Combined with Glactin-3 Had a High Diagnostic Value in Differentiating Benign Thyroid Tumor from PTC}

To further study the diagnostic value of serum lncRNA HOTAIR and Glactin-3 expressions in differentiating benign thyroid tumors from PTC, the ROC curve was used to analyze the diagnostic efficacy of lncRNA HOTAIR, Glactin-3, and their combination. The results demonstrated that the AUC of IncRNA HOTAIR for the diagnosis of PTC was 0.895 , the specificity was $96.00 \%$, and the sensitivity was $80.63 \%(P<0.0001)$ (Figure $2 \mathrm{~A})$. The AUC of Glactin-3 for the diagnosis of PTC was 0.817 , the specificity was $66.67 \%$, and the sensitivity was $78.75 \%$ $(P<0.0001)$ (Figure 2B). The AUC of the combination of IncRNA HOTAIR and Glactin-3 for the diagnosis of PTC was 0.969 , the specificity was $96 \%$, and the sensitivity was $87.50 \%(P<0.0001)$ (Figure $2 \mathrm{C})$. The area difference analysis of AUC showed that the diagnostic efficiency of lncRNA HOTAIR was significantly higher than that of Glactin-3 $(P=0.0153)$, while that of the combination of lncRNA HOTAIR and Glactin-3 was significantly higher than IncRNA HOTAIR $(P=0.0001)$ and Glactin-3 alone $(P<0.0001)$ (Figure 2D). These results indicated that IncRNA HOTAIR combined with Glactin-3 had higher diagnostic efficiency.

\section{Discussion}

The prevalence of thyroid cancer is increasing and PTC is the most common subtype. ${ }^{25}$ Although its characteristics are classically considered as an indolent clinical process with long-term survival rates, some histological variants can have aggressive behaviors and are related to poor clinical outcomes. ${ }^{26}$ Therefore, it is of vital importance to study the mechanism and the diagnostic markers of PTC. Evidence has shown that IncRNA HOTAIR and Glactin-3 play an essential role in PTC. This study found that IncRNA HOTAIR combined with Galectin-3 had a high diagnostic value in differentiating benign thyroid tumor from PTC.

The examination for thyroid includes the blood tests of $\mathrm{TG}-\mathrm{Ab}$ and $\mathrm{TPO}-\mathrm{Ab}{ }^{27}$ Our results demonstrated that there was no significant difference in age, gender, BMI, and TSH level between PTC patients and benign thyroid tumor patients. The levels of TPO-Ab and TG- 
Table 3 Relationship Between IncRNA HOTAIR Expression and Clinical Baseline Characteristics in PTC Patients

\begin{tabular}{|c|c|c|c|c|c|}
\hline \multicolumn{2}{|l|}{ Parameters } & \multirow{3}{*}{$\begin{array}{c}\text { Total } \\
57\end{array}$} & \multicolumn{2}{|c|}{ LncRNA HOTAIR } & \multirow{3}{*}{$\begin{array}{c}P \\
0.409\end{array}$} \\
\hline & & & \multirow{2}{*}{$\begin{array}{c}\begin{array}{c}\text { High } \\
(\mathbf{N}=80)\end{array} \\
31\end{array}$} & \multirow{2}{*}{$\begin{array}{c}\begin{array}{c}\text { Low } \\
(\mathbf{N}=\mathbf{8 0})\end{array} \\
26\end{array}$} & \\
\hline Age(years) & $<55$ & & & & \\
\hline & $\geq 55$ & 103 & 49 & 54 & \\
\hline \multirow[t]{2}{*}{ Gender } & Male & 61 & 35 & 26 & \multirow[t]{2}{*}{0.143} \\
\hline & Female & 99 & 45 & 54 & \\
\hline \multirow[t]{2}{*}{ BMI } & $<24$ & 96 & 45 & 51 & \multirow[t]{2}{*}{0.333} \\
\hline & $\geq 24$ & 64 & 35 & 29 & \\
\hline \multirow{2}{*}{$\begin{array}{l}\text { Tumor size } \\
(\mathrm{cm})\end{array}$} & $<2$ & 75 & 27 & 48 & \multirow[t]{2}{*}{0.001} \\
\hline & $\geq 2$ & 85 & 53 & 32 & \\
\hline \multirow[t]{2}{*}{ Multifocality } & Unifocal & 88 & 45 & 43 & \multirow[t]{2}{*}{0.751} \\
\hline & Multifocal & 72 & 35 & 37 & \\
\hline \multirow[t]{2}{*}{ TNM stage } & I-II & 117 & 50 & 67 & \multirow[t]{2}{*}{0.0042} \\
\hline & III-IV & 43 & 30 & 13 & \\
\hline \multirow[t]{2}{*}{ T stage } & TI-T2 & 83 & 28 & 55 & \multirow[t]{2}{*}{$<0.001$} \\
\hline & T3-T4 & 77 & 52 & 25 & \\
\hline \multirow{2}{*}{$\begin{array}{l}\text { Lymph node } \\
\text { metastasis }\end{array}$} & No & 66 & 20 & 46 & \multirow[t]{2}{*}{$<0.001$} \\
\hline & $\mathrm{NI}$ & 94 & 60 & 34 & \\
\hline \multirow{2}{*}{$\begin{array}{l}\text { Distant } \\
\text { metastasis }\end{array}$} & MI & 11 & 6 & 5 & \multirow[t]{2}{*}{0.755} \\
\hline & Mo & 149 & 74 & 75 & \\
\hline \multicolumn{2}{|l|}{ TSH (mU/L) } & $\begin{array}{c}3.25 \pm \\
0.71\end{array}$ & $\begin{array}{l}3.17 \pm \\
0.69\end{array}$ & $\begin{array}{c}3.33 \pm \\
0.73\end{array}$ & 0.156 \\
\hline \multicolumn{2}{|l|}{ TPO-Ab (U/L) } & $\begin{array}{c}3.78 \pm \\
0.73\end{array}$ & $\begin{array}{c}3.91 \pm \\
0.75\end{array}$ & $\begin{array}{c}3.65 \pm \\
0.69\end{array}$ & $<0.024$ \\
\hline \multicolumn{2}{|l|}{ TG-Ab (U/L) } & $\begin{array}{c}3.55 \pm \\
0.69\end{array}$ & $\begin{array}{c}3.69 \pm \\
0.67\end{array}$ & $\begin{array}{c}3.41 \pm \\
0.68\end{array}$ & 0.010 \\
\hline
\end{tabular}

$\mathrm{Ab}$ in PTC patients were significantly higher than those in patients with benign thyroid tumor. Consistently, TPO-Ab and TG-Ab levels are elevated in the serum of PTC patients. ${ }^{28}$ In conclusion, PTC patients and benign thyroid tumor patients showed different clinical indexes.

LncRNA HOTAIR is coded from homebox $\mathrm{C}$ gene locus and is involved in the progression and development of a variety of malignancies. ${ }^{29}$ LncRNA HOTAIR plays a crucial role in PTC. ${ }^{13}$ Galectin-3 is identified to be a histological marker of thyroid cancer. ${ }^{16}$ However,
Table 4 Relationship Between Glactin-3 Expression and Clinical Baseline Characteristics in PTC Patients

\begin{tabular}{|c|c|c|c|c|c|}
\hline \multicolumn{2}{|l|}{ Parameters } & \multirow{3}{*}{$\begin{array}{c}\text { Total } \\
57\end{array}$} & \multicolumn{2}{|c|}{ LncRNA HOTAIR } & \multirow{3}{*}{$\begin{array}{c}P \\
0.137\end{array}$} \\
\hline & & & $\begin{array}{c}\text { High } \\
(\mathrm{N}=\mathbf{8 0})\end{array}$ & $\begin{array}{c}\text { Low } \\
(\mathrm{N}=\mathbf{8 0})\end{array}$ & \\
\hline \multirow[t]{2}{*}{ Age(years) } & $<55$ & & 33 & 24 & \\
\hline & $\geq 55$ & 103 & 47 & 56 & \\
\hline \multirow[t]{2}{*}{ Gender } & Male & 61 & 32 & 29 & \multirow[t]{2}{*}{0.625} \\
\hline & Female & 99 & 48 & 51 & \\
\hline \multirow[t]{2}{*}{ BMI } & $<24$ & 96 & 46 & 50 & \multirow[t]{2}{*}{0.519} \\
\hline & $\geq 24$ & 64 & 34 & 30 & \\
\hline \multirow[t]{2}{*}{ Tumor size $(\mathrm{cm})$} & $<2$ & 75 & 26 & 49 & \multirow{2}{*}{$\begin{array}{c}< \\
0.001\end{array}$} \\
\hline & $\geq 2$ & 85 & 54 & 31 & \\
\hline \multirow[t]{2}{*}{ Multifocality } & Unifocal & 88 & 41 & 47 & \multirow[t]{2}{*}{0.340} \\
\hline & Multifocal & 72 & 39 & 33 & \\
\hline \multirow[t]{2}{*}{ TNM stage } & $1-11$ & 117 & 52 & 65 & \multirow[t]{2}{*}{$0.02 C$} \\
\hline & III-IV & 43 & 28 & 15 & \\
\hline \multirow[t]{2}{*}{ T stage } & TI-T2 & 83 & 30 & 53 & \multirow{2}{*}{$\begin{array}{c}< \\
0.00\end{array}$} \\
\hline & T3-T4 & 77 & 50 & 27 & \\
\hline \multirow{2}{*}{$\begin{array}{l}\text { Lymph node } \\
\text { metastasis }\end{array}$} & No & 66 & 20 & 46 & \multirow{2}{*}{$\begin{array}{c}< \\
0.001\end{array}$} \\
\hline & $\mathrm{NI}$ & 94 & 60 & 34 & \\
\hline \multirow{2}{*}{$\begin{array}{l}\text { Distant } \\
\text { metastasis }\end{array}$} & MI & 11 & 6 & 5 & \multirow[t]{2}{*}{0.75} \\
\hline & Mo & 149 & 66 & 83 & \\
\hline \multicolumn{2}{|l|}{$\mathrm{TSH}(\mathrm{mU} / \mathrm{L})$} & $\begin{array}{c}3.25 \pm \\
0.71\end{array}$ & $\begin{array}{c}3.24 \pm \\
0.75\end{array}$ & $\begin{array}{c}3.26 \pm \\
0.67\end{array}$ & 0.859 \\
\hline \multicolumn{2}{|l|}{ TPO-Ab (U/L) } & $\begin{array}{c}3.78 \pm \\
0.73\end{array}$ & $\begin{array}{c}3.89 \pm \\
0.73\end{array}$ & $\begin{array}{c}3.67 \pm \\
0.71\end{array}$ & 0.055 \\
\hline \multicolumn{2}{|l|}{ TG-Ab (U/L) } & $\begin{array}{c}3.55 \pm \\
0.69\end{array}$ & $\begin{array}{c}3.56 \pm \\
0.71\end{array}$ & $\begin{array}{c}3.54 \pm \\
0.67\end{array}$ & 0.855 \\
\hline
\end{tabular}

the mechanism of lncRNA HOTAIR and Galectin-3 affecting PTC clinical features remains unknown. Our results showed that serum expressions of lncRNA HOTAIR and Galectin-3 in PTC patients were significantly higher than those in patients with benign thyroid tumor. Consistently, the expression of HOTAIR in PTC tissues is higher than that in normal tissues. ${ }^{29}$ Briefly, lncRNA HOTAIR and Glactin-3 were highly expressed in PTC. Then, we analyzed the correlation between IncRNA HOTAIR/Glactin-3 and the clinical indicators, and found that patients with high lncRNA HOTAIR 
A

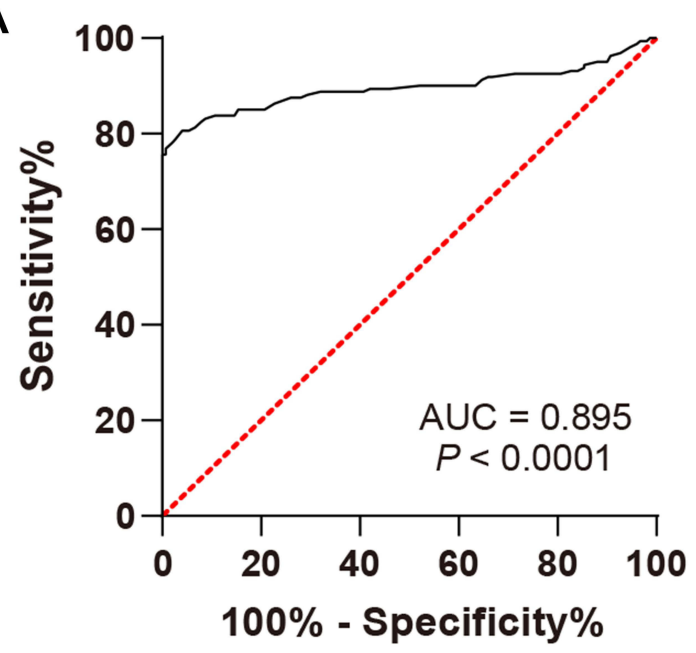

C

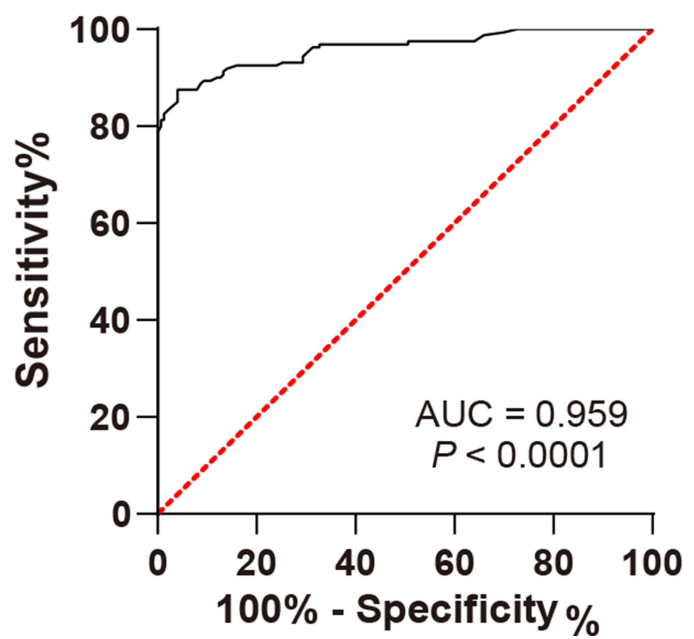

B

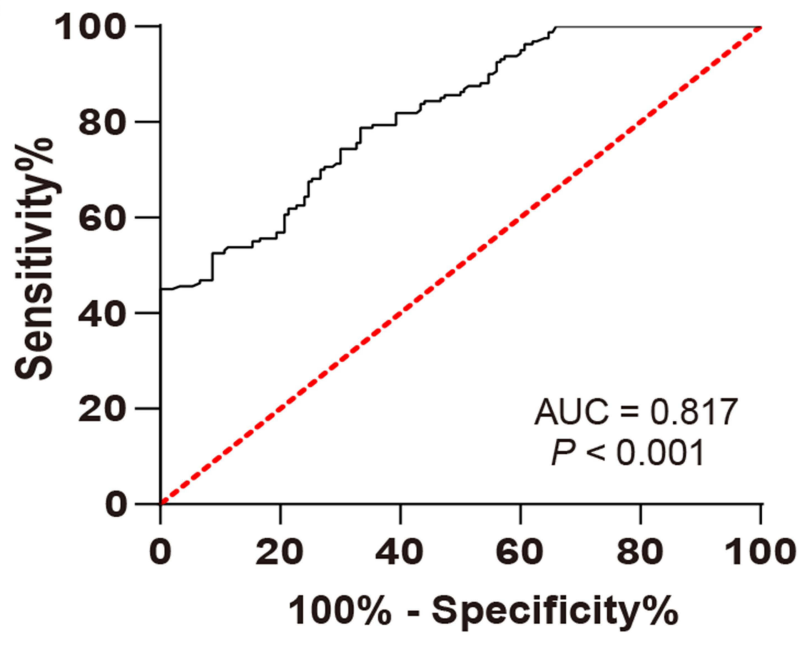

D

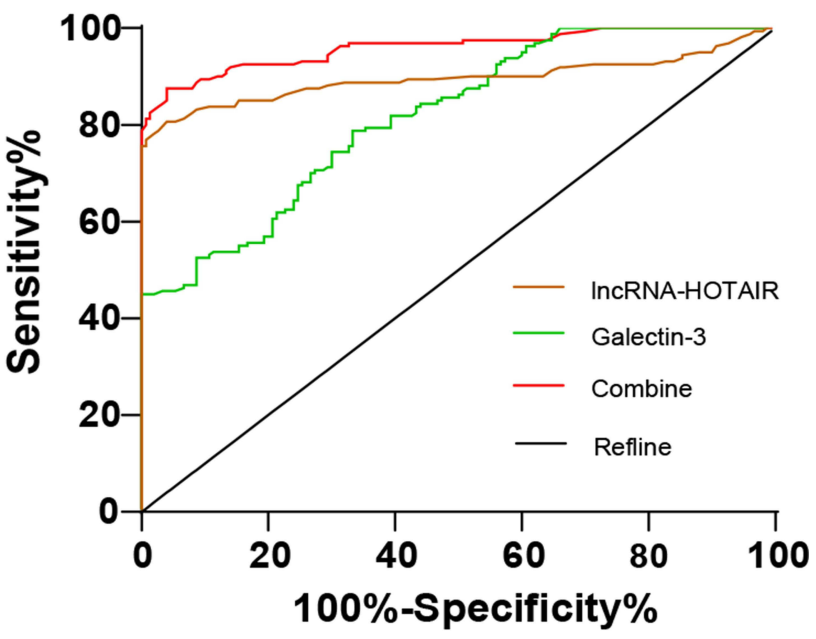

Figure 2 LncRNA HOTAIR combined with Glactin-3 had higher diagnostic efficiency in differentiating benign thyroid tumor from PTC. (A) ROC curve analysis of diagnostic efficacy of IncRNA HOTAIR; (B) ROC curve analysis of diagnostic efficacy of Glactin-3; (C) ROC curve analysis of diagnostic efficacy of the combination of LncRNA HOTAIR and Glactin-3; (D) ROC curve was used to compare the diagnostic efficacy of IncRNA HOTAIR, Glactin-3, and their combination.

expression had a larger tumor diameter, more advanced TNM stage, higher $\mathrm{T}$ stage, more lymph node metastasis, and higher TPO-Ab and TG-Ab expressions, but with no correlation with age, gender, BMI, multifocality, distant metastasis, and TSH expression. Another study also manifested consistent findings that lncRNA HOTAIR expression is correlated with the clinical indicators including pathological stage and lymph node metastasis in patients with PTC. ${ }^{12,30}$ Moreover, patients with high Glactin-3 expression were correlated with larger tumor diameter, more advanced TNM stage, and more lymph node metastasis, but with no correlation with age, gender, BMI, multifocality, $\mathrm{T}$ stage, distant metastasis, TSH, TPO-Ab, and TG-Ab expressions. There is a higher rate of positive Galectin-3 expression in thyroid cancer patients with lymph node metastasis. ${ }^{31}$
Altogether, lncRNA HOTAIR and Glactin-3 were associated with clinical parameters of PTC patients.

LncRNA-HOTAIR in tissue samples of patients with PTC has been recommended for PTC prognosis. ${ }^{14}$ Galectin-3 is recognized as a tissue marker of PTC and has good specificity in differentiating thyroid cancer. $^{16,18,32}$ However, there are few studies on the clinical significance of serum Galectin-3 value in PTC. The clinical significance of lncRNA-HOTAIR serum value and its combination with Galectin-3 in PTC has not been reported. To further study the clinical significance of IncRNA HOTAIR/Glactin-3 expressions in PTC, we analyzed the diagnostic efficacy of IncRNA HOTAIR or/and Glactin-3. Our results demonstrated that the AUC of IncRNA HOTAIR for PTC diagnosis was 0.895 , the specificity was $96 \%$, and the sensitivity was $80.63 \%$. The 
AUC of Glactin-3 for PTC diagnosis was 0.817 , the specificity was $66.67 \%$, and the sensitivity was $78.75 \%$. The AUC of the combination of lncRNA HOTAIR and Glactin-3 for PTC diagnosis was 0.969 , the specificity was $96 \%$, and the sensitivity was $87.50 \%$. These results suggested that the AUC of IncRNA HOTAIR was significantly higher than that of Glactin-3, while the AUC of the combination of lncRNA HOTAIR and Glactin-3 was significantly higher than that of IncRNA HOTAIR and Glactin-3 alone. In brief, IncRNA HOTAIR combined with Glactin-3 had higher diagnostic efficiency for PTC.

In summary, this study supported that the combination of IncRNA HOTAIR and Glactin-3 had higher diagnostic efficiency, which provided some reference for the clinical diagnosis of PTC. We retrospectively analyzed the relationship between lncRNA HOTAIR, Glactin-3, and the clinical features of PTC in a relatively small cohort. Further study on the molecular mechanism of overexpression of lncRNA HOTAIR and Glactin-3 would help to verify our results. In this study, we only analyzed that the detection of lncRNA HOTAIR combined with Glactin-3 had higher diagnostic efficiency for PTC. The combined diagnostic value of IncRNA HOTAIR and other possible factors, and the combined diagnostic value of Glactin-3 and other differentially expressed lncRNAs, remain to be further explored.

\section{Data Sharing Statement}

All the data generated or analyzed during this study are included in this published article.

\section{Author Contributions}

All authors made a significant contribution to the work reported, whether that is in the conception, study design, execution, acquisition of data, analysis, and interpretation, or in all these areas; took part in drafting, revising, or critically reviewing the article; gave final approval of the version to be published; have agreed on the journal to which the article has been submitted; and agree to be accountable for all aspects of the work.

\section{Funding}

There is no funding to report.

\section{Disclosure}

The authors declared that they have no competing interests.

\section{References}

1. Pellegriti G, Frasca F, Regalbuto C, Squatrito S, Vigneri R. Worldwide increasing incidence of thyroid cancer: update on epidemiology and risk factors. J Cancer Epidemiol. 2013;2013:965212. doi:10.1155/2013/965212

2. Vigneri R, Malandrino P, Vigneri P. The changing epidemiology of thyroid cancer: why is incidence increasing? Curr Opin Oncol. 2015;27(1):1-7. doi:10.1097/CCO.0000000000000148

3. Carcangiu ML, Zampi G, Rosai J. Poorly differentiated ("insular") thyroid carcinoma. A reinterpretation of Langhans' "wuchernde Struma". Am J Surg Pathol. 1984;8(9):655-668. doi:10.1097/ 00000478-198409000-00005

4. Duskova J. Known pitfalls of the thyroid neoplasm diagnostics in the view of the new (2004) WHO Classification. Cesk Patol. 2006;42 (1):24-28

5. Kondo T, Ezzat S, Asa SL. Pathogenetic mechanisms in thyroid follicular-cell neoplasia. Nat Rev Cancer. 2006;6(4):292-306. doi: $10.1038 / \mathrm{nrc} 1836$

6. LiVolsi VA, Asa SL. The demise of follicular carcinoma of the thyroid gland. Thyroid. 1994;4(2):233-236. doi:10.1089/ thy.1994.4.233

7. Mazzaferri EL. Papillary thyroid carcinoma: factors influencing prognosis and current therapy. Semin Oncol. 1987;14(3):315-332.

8. McCaffrey TV, Lipton RJ. Thyroid carcinoma invading the upper aerodigestive system. Laryngoscope. 1990;100(8):824-830. doi:10.1288/00005537-199008000-00006

9. Prasad ML, Huang Y, Pellegata NS, de la Chapelle A, Kloos RT. Hashimoto's thyroiditis with papillary thyroid carcinoma (PTC)-like nuclear alterations express molecular markers of PTC. Histopathology. 2004;45(1):39-46. doi:10.1111/j.1365-2559.2004.01876.x

10. Zhang X, Hong R, Chen W, Xu M, Wang L. The role of long noncoding RNA in major human disease. Bioorg Chem. 2019;92:103214. doi:10.1016/j.bioorg.2019.103214

11. Tan J, Liu L, Zuo Z, et al. Overexpression of novel long intergenic noncoding RNA LINC02454 is associated with a poor prognosis in papillary thyroid cancer. Oncol Rep. 2020;44(4):1489-1501.

12. Wu L, Shi Y, Liu B, Zhao M. Expression of IncRNA-HOTAIR in the serum of patients with lymph node metastasis of papillary thyroid carcinoma and its impact. Oncol Lett. 2020;20(1):907-913. doi:10.3892/ol.2020.11620

13. Xia F, Xia W, Yu X. LncRNA HOTAIR influences the growth, migration, and invasion of papillary thyroid carcinoma via affection on the miR-488-5p/NUP205 axis. Technol Cancer Res Treat. 2020;19:1533033820962125. doi:10.1177/1533033820962125

14. Jiang Y, Wang J, Chen J, Wang J, Xu J. Construction and analysis of an aberrant IncRNA-miRNA-mRNA network associated with papillary thyroid cancer. Medicine. 2020;99(45):e22705. doi:10.1097/ MD.0000000000022705

15. Nangia-Makker P, Hogan V, Raz A. Galectin-3 and cancer stemness. Glycobiology. 2018;28(4):172-181. doi:10.1093/glycob/cwy001

16. De Rose F, Braeuer M, Braesch-Andersen S, et al. Galectin-3 targeting in thyroid orthotopic tumors opens new ways to characterize thyroid cancer. J Nucl Med. 2019;60(6):770-776. doi:10.2967/ jnumed.118.219105

17. Isic T, Savin S, Cvejic D, et al. Serum Cyfra 21.1 and galectin-3 protein levels in relation to immunohistochemical cytokeratin 19 and galectin-3 expression in patients with thyroid tumors. $J$ Cancer Res Clin Oncol. 2010;136(12):1805-1812. doi:10.1007/s00432-010-0838-3

18. Makki FM, Taylor SM, Shahnavaz A, et al. Serum biomarkers of papillary thyroid cancer. J Otolaryngol Head Neck Surg. 2013;42:16. doi:10.1186/1916-0216-42-16

19. Chang L, Guo R, Yuan Z, Shi H, Zhang D. LncRNA HOTAIR regulates CCND1 and CCND2 expression by sponging miR-206 in ovarian cancer. Cell Physiol Biochem. 2018;49(4):1289-1303. doi: $10.1159 / 000493408$ 
20. $\mathrm{Xu} \mathrm{Y,} \mathrm{Hu} \mathrm{J,} \mathrm{Zhang} \mathrm{Y,} \mathrm{et} \mathrm{al.} \mathrm{LncRNA} \mathrm{HOTAIR} \mathrm{modulates} \mathrm{the}$ expression of OATP1B1 in HepG2 cells by sponging miR-206/miR613. Xenobiotica. 2020;50(12):1494-1500. doi:10.1080/ 00498254.2020.1777484

21. Zhang J, Li N, Fu J, Zhou W. Long noncoding RNA HOTAIR promotes medulloblastoma growth, migration and invasion by sponging miR-1/miR-206 and targeting YY1. Biomed Pharmacother. 2020;124:109887. doi:10.1016/j.biopha.2020.109887

22. Liu F, Yin R, Chen X, et al. Over-expression of miR-206 decreases the Euthyrox-resistance by targeting MAP4K3 in papillary thyroid carcinoma. Biomed Pharmacother. 2019;114:108605. doi:10.1016/j. biopha.2019.108605

23. Tam S, Boonsripitayanon M, Amit M, et al. Survival in differentiated thyroid cancer: comparing the AJCC cancer staging seventh and eighth editions. Thyroid. 2018;28(10):1301-1310. doi:10.1089/ thy. 2017.0572

24. Schmittgen TD, Livak KJ. Analyzing real-time PCR data by the comparative C(T) method. Nat Protoc. 2008;3(6):1101-1108. doi:10.1038/nprot.2008.73

25. Bergdorf K, Ferguson DC, Mehrad M, Ely K, Stricker T, Weiss VL. Papillary thyroid carcinoma behavior: clues in the tumor microenvironment. Endocr Relat Cancer. 2019;26(6):601-614. doi:10.1530/ERC-19-0074

26. Canberk S, Montezuma D, Ince U, et al. Variants of papillary thyroid carcinoma: an algorithmic cytomorphology-based approach to cytology specimens. Acta Cytol. 2020;64(4):288-298. doi:10.1159/ 000503576
27. Soh SB, Aw TC. Laboratory testing in thyroid conditions - pitfalls and clinical utility. Ann Lab Med. 2019;39(1):3-14. doi:10.3343/ alm.2019.39.1.3

28. Hwang S, Shin DY, Kim EK, et al. Focal lymphocytic thyroiditis nodules share the features of papillary thyroid cancer on ultrasound. Yonsei Med J. 2015;56(5):1338-1344. doi:10.3349/ ymj.2015.56.5.1338

29. Zhu H, Lv Z, An C, et al. Onco-lncRNA HOTAIR and its functional genetic variants in papillary thyroid carcinoma. Sci Rep. 2016;6:31969. doi:10.1038/srep31969

30. Chen X, Jin J, Zheng L, Sheng Y, Sun J. Correlations of HOTAIR expression with pathological stage, CT characteristics and prognosis of patients with papillary thyroid carcinoma. J BUON. 2021;26 (1):259-265.

31. Liu Y, Meng H, Xu S, Qi X. Galectins for diagnosis and prognostic assessment of human diseases: an overview of meta-analyses. Med Sci Monit. 2020;26:e923901. doi:10.12659/MSM.923901

32. Cho H, Kim JY, Oh YL. Diagnostic value of HBME-1, CK19, Galectin 3, and CD56 in the subtypes of follicular variant of papillary thyroid carcinoma. Pathol Int. 2018;68(11):605-613. doi:10.1111/ pin. 12729

\section{Publish your work in this journal}

Cancer Management and Research is an international, peer-reviewed open access journal focusing on cancer research and the optimal use of preventative and integrated treatment interventions to achieve improved outcomes, enhanced survival and quality of life for the cancer patient.
The manuscript management system is completely online and includes a very quick and fair peer-review system, which is all easy to use. Visit http://www.dovepress.com/testimonials.php to read real quotes from published authors. 